\title{
Mechanism of Spontaneous Rhythmic Contraction in Isolated Rat Large Artery
}

\author{
Naoki Hayashida, ${ }^{* * *}$ Katsuji OKuI, ${ }^{*}$ and Yasuichiro FukudA ${ }^{* *}$ \\ *Department of Surgery I and **Department of Physiology II, \\ School of Medicine, Chiba University, Chiba, 280 Japan
}

\begin{abstract}
The mechanism of spontaneous contraction of vascular smooth muscles in the elastic artery was studied in a ring-shaped preparation isolated from the rat aorta. The observation of small changes in vascular tension with a high gain AC amplification of tension signal provided a reliable detection of spontaneous contractions. The spontaneous rhythmic contraction (RC) occurred consistently in the preparation taken from the thoracic aorta without external stimuli. The RC (frequency, 5-20 cycle/min; amplitude, $10-100 \mathrm{mg}$ ) was accompanied with small oscillatory changes in the membrane potential $(2-5 \mathrm{mV}$, peak to peak). A reduction in temperature (below $30^{\circ} \mathrm{C}$ ) or superfusing the preparation with $\mathrm{Ca}$-free solution inhibited the generation of RC. Ca-entry blockers (verapamil and nifedipine) also inhibited the RC. The cessation of RC by these procedures reduced the vascular tension by about $40 \%$ of control baseline tension. The application of adrenergic blockers had little effect on the pattern of $\mathrm{RC}$ and on the vascular tension. The results suggest that the RC is generated by a synchronization of electrical and mechanical activities in relatively small groups of smooth muscle cells, which depends upon the temperature and requires the Ca-entry into the cells. The process of initiation of spontaneous $\mathrm{RC}$ in the rat aorta was discussed.
\end{abstract}

Key words: vascular smooth muscle, rat aorta, spontaneous rhythmic contraction, membrane potential, $\mathrm{Ca}$.

The contraction of vascular smooth muscles alters the vessel diameter and regulates the vascular "tone." The contractile behavior or the pattern of contraction, however, differs between various segments of vascular tree. Many previous studies have shown that some isolated vessels, such as the portal vein of the rat, subcutaneous artery of the dog, and bovine mesenteric vein, generate spontaneous rhythmic contractions (FunAKI and BOHR, 1964; JOHANSSON and BOHR, 1966; RODDIE and SCOTT, 1969). These rhythmic contractions are due to synchronization

Received for publication April 23, 1986 
of electrical and mechanical activities of many smooth muscle cells. In the large elastic arteries, on the other hand, spontaneous contractions occur only occasionally or can be initiated by application of chemical stimuli (high potassium, catecholamines, or low magnesium) (BIAmino and KruCKenberg, 1969; Altura and Altura, 1974; Altura et al., 1980). Although the media of elastic artery contains plenty of smooth muscle cells, it is not certain whether these smooth muscles are inherently incapable of generating spontaneous rhythmic contractions. Furthermore, the mechanism of spontaneous contractions, if any, in the elastic arteries, has not been studied systematically. The present experiments aimed to observe the spontaneous contractions in the large conduit artery and to assess their generating mechanism and their contribution to the maintenance of vascular tension. The results showed that the large elastic artery of the rat is capable of initiating spontaneous rhythmic contractions.

\section{METHODS}

The aorta and its branches were isolated from the male Wistar rat weighing 200-300 g under pentobarbital anesthesia $(50 \mathrm{mg} / \mathrm{kg}$, i.p.). Ring-shaped vascular preparations (1-2 mm diameter, $2-4 \mathrm{~mm}$ width) were obtained from various regions of the large artery by transverse section and were placed in an incubation chamber (6 $\mathrm{ml}$ volume) (Fig. 1). The preparation was continuously superfused $(6 \mathrm{ml} / \mathrm{min})$ with the physiological salt solution (modified Tyrode solution) having the following composition in mM: $\mathrm{NaCl} 120, \mathrm{KCl} 5.0, \mathrm{CaCl}_{2} 1.5, \mathrm{MgCl}_{2} 0.5, \mathrm{NaH}_{2} \mathrm{PO}_{4} 1.0$, $\mathrm{NaHCO}_{3} 24.0$, and glucose 10.0. The solution was bubbled with a gas mixture containing $5 \% \mathrm{CO}_{2}$ and $95 \% \mathrm{O}_{2}$ in a reservoir bottle ( $\left.\mathrm{pH} 7.40\right)$. The temperature of the solution was maintained at $37^{\circ} \mathrm{C}$ except when the effect of low temperature on the vascular contraction was examined. In the incubation chamber the preparation was suspended between two stainless steel rods, one for fixating the preparation and the other for connecting it to a strain-gauge transducer via a synthetic surgical thread (polyester fiber, TI-CRON ${ }^{\circledR}$ No. 5-0 or 6-0, Davis Geck), to registrate the isometric circular tension of the arterial segment (Fig. 1). The preparation was usually stretched transversely to apply baseline tension of about $200-400 \mathrm{mg}$. In some experiments the level of baseline tension was varied between 0 and $400 \mathrm{mg}$. The application of stretch tension higher than $500 \mathrm{mg}$ was avoided because such a high baseline tension was not maintained constant but it was decreased gradually during the experiment. The output from a tension amplifier (carrier amplifier) was fed not only directly into a pen recorder (DC mode) for monitoring the baseline tension but also into a band pass filter (AC) amplifier (band width $0.05-10 \mathrm{~Hz}$ ) for monitoring small changes in vascular tension due to spontaneous contraction. The noise level of the system for measuring the mechanical activity was reduced to about $1 \mathrm{mg}$ (peak to peak) by using double mechanical shock absorbers (rubber pads), by the use of an appropriate band pass filter amplifier (see above) and by preventing the preparation-transducer system from touching directly with the wall of incubation 


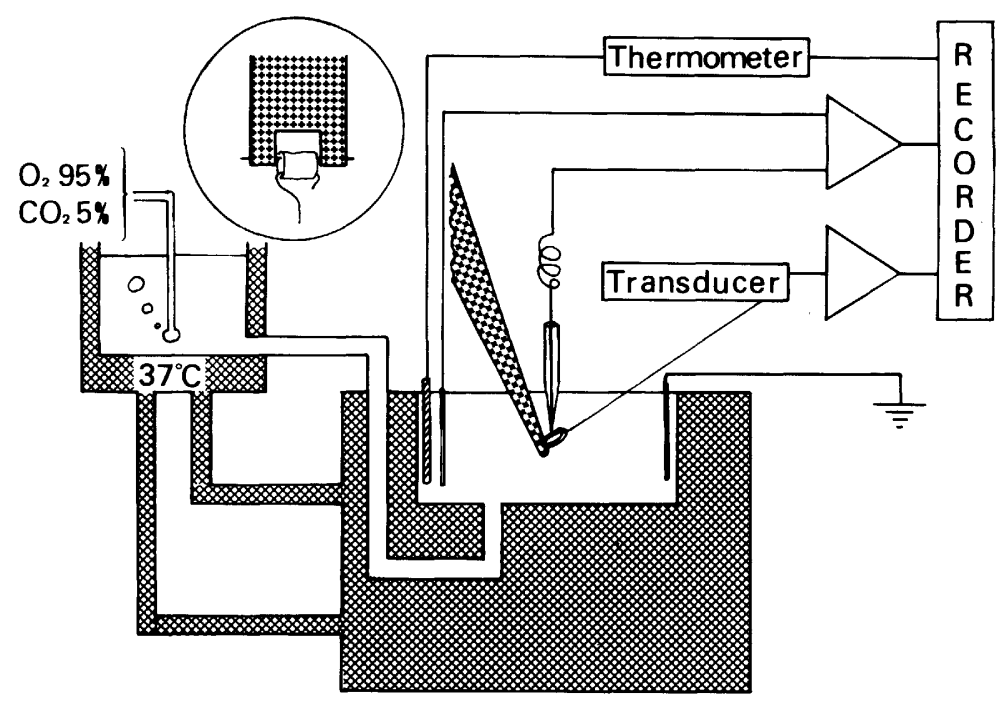

Fig. 1. Schematic illustration of experimental setup. A ring-shaped vascular preparation was placed in the incubation chamber. The fixation and connection of preparation to a strain-gauge transducer by stainless steel rods and a thread are shown in the enlarged figure within the circle. The cross-hatched area was filled by circulating water $\left(37^{\circ} \mathrm{C}\right)$.

chamber which was connected to a circulating pump (Fig. 1).

The membrane potential of a single muscle cell was recorded by a conventional $3 \mathrm{M}-\mathrm{KCl}$ filled floating glass microelectrode (resistance, $10-20 \mathrm{M} \Omega$ ). The microelectrode was inserted into the muscular layer from the adventitial surface or from the intimal layer. The transmembrane potential was monitored on an oscilloscope and was registered on a pen recorder together with mechanical activities and a temperature of the superfusing solution in the incubation chamber.

In some other experiments, effects on mechanical activities of a low temperature, nominally $\mathrm{Ca}$-free solution or Ca-entry blockers (verapamil $\mathrm{HCl}$, Eisai; nifedipine, Bayer) were investigated. Several adrenergic blocking agents (phentolamine mesylate, Ciba; prazosin $\mathrm{HCl}$, Pfizer; yohimbine $\mathrm{HCl}$, Wako; propranolol $\mathrm{HCl}$, ICI) were also used to clarify whether the endogenous catecholamine contributed to the generation of spontaneous contractions. A total amount of $0.1-$ $0.3 \mathrm{ml}$ drug solution was injected for a period 1-3 min into a side tube through which the superfusing solution flowed into the incubation chamber. An approximate mean concentration of a drug in the incubation chamber was calculated. In some experiments, the physiological salt solution containing a known concentration of a drug was flowed into the incubation chamber. When nifedipine was used, the bath and solutions were protected from light. Circulating water which surrounded the incubation chamber and reservoir bottle was cooled by ice when the effects of 
low temperature on the pattern of spontaneous contraction were studied.

\section{RESULTS}

\section{Spontaneous rhythmic contraction in the rat large artery}

The preparations isolated from the aorta and other large arteries showed spontaneous rhythmic contractions (RC) (Fig. 2). The typical RC was more consistently seen in the preparation taken from the thoracic aorta (including the ascending aorta and aortic arch) than that from more distal aorta or from some aortic branches (common carotid and common iliac arteries). The present experiment was, therefore, performed on the preparation obtained from the thoracic aorta. The RC was a relatively regular contraction usually seen in both the DC (low gain) and $\mathrm{AC}$ (high gain) tension registrations. The frequency of $\mathrm{RC}$ ranged from 4.7 to $19.6 \mathrm{cycle} / \mathrm{min}(11.9 \pm 4.9 \mathrm{cycle} / \mathrm{min}$, mean \pm S.D., $n=20)$. When the baseline tension was increased up to about $400 \mathrm{mg}$ by increasing passive stretch, the frequency remained fairly constant in the static phase of stretch (Fig. 2). Changes in the frequency in a dynamic phase of stretch were not systematically studied because a transient change in the frequency (i.e., increase) during or shortly after increasing the passive stretch was slight and it occurred only occasionally. The amplitude of $\mathrm{RC}$, on the other hand, was increased with increasing the stretch load (Fig. 2). Generally the amplitude of RC (10-100 $\mathrm{mg}$ at a baseline steady state tension of about $300 \mathrm{mg}$ ) correlated inversely with the frequency, i.e., the higher the frequency,

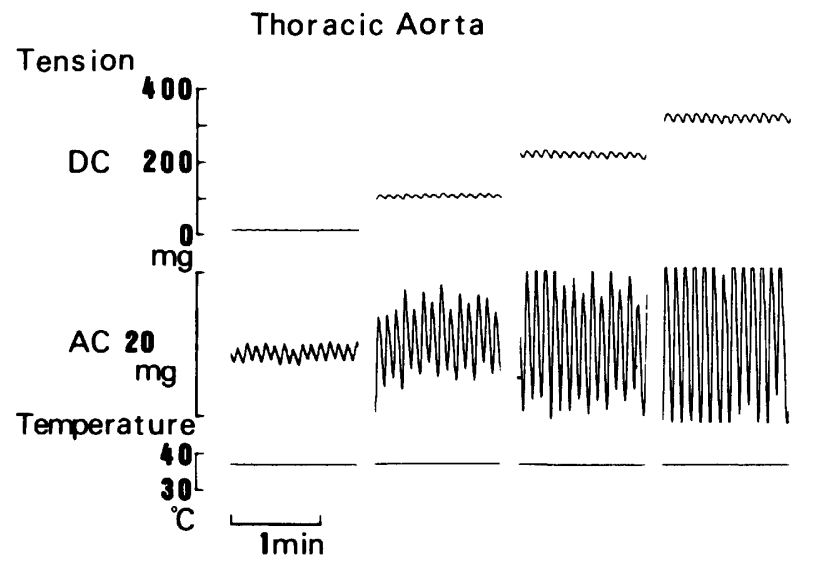

Fig. 2. A typical example of spontaneous rhythmic contraction (RC) in the rat large artery (thoracic aorta). The $\mathrm{RC}$ at various levels of baseline tension (steady state condition) by increasing passive stretch is shown. DC, baseline vascular tension measured with low gain amplification in the DC mode; AC, tension registration with high gain amplification in the AC mode. These abbreviations are the same in following similar figures. Registration of changes in tension (AC mode) was partly saturated on a pen recorder. 


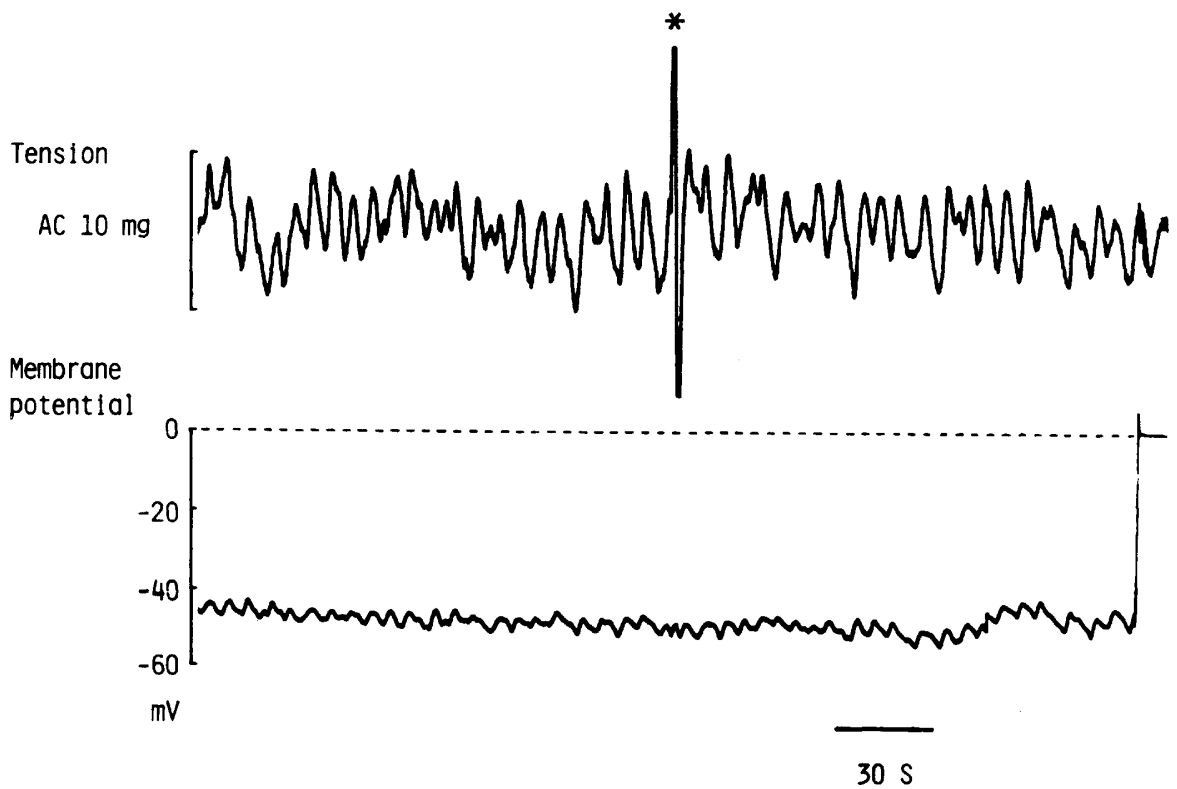

Fig. 3. Relationship between membrane potential and spontaneous rhythmic contraction (RC) in the rat thoracic aorta. Although the majority of changes in tension (RC) accompanied the small and slow depolarization in the recorded cell, a few contractions occurred without accompanying the cellular depolarization. The asterisk indicates the time when the preparation was passively stretched to increase the tension then quickly returned to previous level. The potential recording was stable though artificial changes in tension (large swings in AC mode registration) were given. The tension registration only in the AC mode is shown. The baseline tension (DC mode) was $300 \mathrm{mg}$.

the lower the amplitude. As shown in Fig. 2, the RC was clearly seen in the AC mode registration when it was not observed in low gain $\mathrm{DC}$ tension registration at a minimum passive stretch less than $20 \mathrm{mg}$.

\section{Changes in membrane potential}

The recording microelectrode was inserted into the smooth muscle layer from the adventitia or in a few cases from the intimal layer. The entry into the cell was identified by a sudden appearance of negative potentials of about $20-50 \mathrm{mV}$. Withdrawal or further penetration of the electrode abruptly abolished this negative potential. An important criterion for the successful recording was that the electrical potential was not shifted by rapid manual alteration of passive stretch tension (within 2-3 s) (Fig. 3, asterisk). This indicated that the changes in potential were not artifacts due to mechanical movements of tissue. The average membrane potential of the cells in the thoracic aorta was $-33.2 \pm 9.3 \mathrm{mV}$ (mean \pm S.D., $n=44$ ). A continuous stable recording fulfilling the above criterion could be made only in 7 


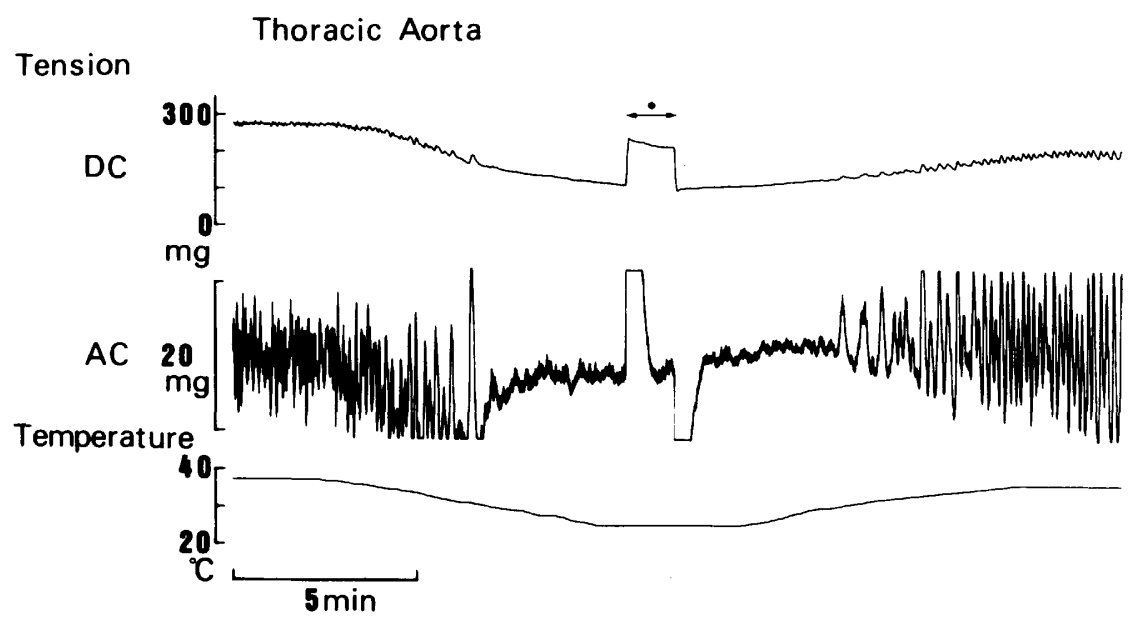

Fig. 4. Effect of low temperature on spontaneous rhythmic contraction in the thoracic aorta. During the period indicated by the arrow with dot, the preparation was passively stretched to restore the baseline tension (large artifactual changes in tension in the AC mode). Small irregular changes in tension (AC mode) remained after the rhythmic contraction disappeared at low temperature. The rhythmic contraction was not initiated by passive increase in stretch tension in low temperature. Registration of changes in tension ( $\mathrm{AC}$ mode) is partly saturated on a pen recorder.

out of 90 cases of RC. In these 7 cases the RC was accompanied by small oscillatory changes in membrane potential $(2-5 \mathrm{mV}$, peak to peak). The synchronization between each contraction and cellular depolarization was almost complete in 2 cases. However, in 5 other cases, only a few individual contractions occurred without accompanying the potential change in the recorded cell although the majority of contractions coincided with small depolarization (Fig. 3). Observations on an oscilloscope revealed that the potential was not associated with rapid action potentials or spikes. Although similar small changes in potential were recorded in other cases, the potentials from these cells were altered also by manual alteration of passive stretch so that they were deleted from analysis.

\section{Effects of low temperature}

The frequency of $\mathrm{RC}$ and the vascular tension were decreased with a reduction in temperature of superfusing solution from 37 to below $30^{\circ} \mathrm{C}$ (average rate of reduction in temperature, $2^{\circ} \mathrm{C} / \mathrm{min}$ ) (Fig. 4). The $\mathrm{RC}$ ceased at about $30^{\circ} \mathrm{C}$, which was accompanied with a reduction in tension by $45 \pm 14 \%($ mean \pm S.D., $n=16)$ of control baseline tension $\left(37^{\circ} \mathrm{C}\right)$. In such low temperatures, the RC could not reappear by an artificial restoration of tension due to an increased passive stretch (Fig. 4, dot). This indicated that the reduction in the baseline tension itself did not inhibit the generation of $\mathrm{RC}$ but the inhibition of $\mathrm{RC}$ by low temperature might 


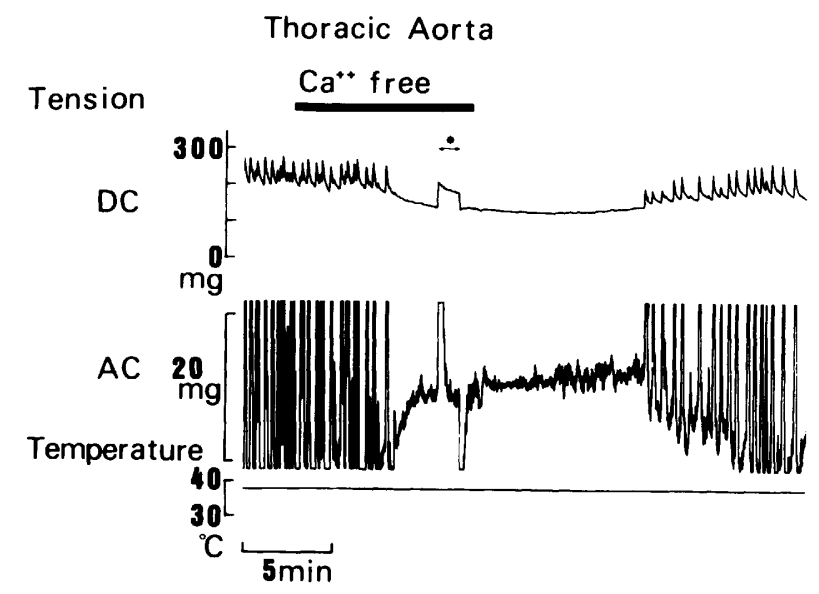

Fig. 5. Effect of Ca-free solution on spontaneous rhythmic contraction in the thoracic aorta. Ca-free solution was applied for a period of time indicated by the heavy horizontal bar. During the period shown by the arrow with dot, the preparation was passively stretched to restore the baseline tension (large artifactual changes in tension in the AC mode). Registration of changes in tension (AC mode) is partly saturated on a pen recorder.

have reduced the baseline tension. As demonstrated in Fig. 4, small irregular changes in tension (2-8 mg) were always seen in the AC mode registration after the $\mathrm{RC}$ had ceased. The amplitude of this remaining small mechanical activity was larger than that of the noise level of the tension measuring system $(1 \mathrm{mg})$. Furthermore the preparations which were killed by alcohol fixation or severely deteriorated preparations showed neither the RC nor such small irregular mechanical activities. Although we did not analyze it systematically, this small mechanical activity seemed to originate in the vascular tissue. The small irregular activity was relatively refractory to low temperature and was abolished by extremely low temperature (below $10^{\circ} \mathrm{C}$ ) only.

\section{Effects of Ca-free solution and Ca-entry blockers}

Superfusing the preparation with nominally $\mathrm{Ca}$-free solution resulted in a decrease in the frequency of RC and consequent decline in tension (Fig. 5). The magnitude of reduction in tension by cessation of RC was $34 \pm 22 \%$ (mean \pm S.D., $n=6$ ) of control baseline tension (normal solution). The $\mathrm{RC}$ recovered completely after switching the superfusing solution from $\mathrm{Ca}$-free to a normal one. The application of verapamil (average concentration in the incubation chamber, above $5.2 \times 10^{-6} \mathrm{M}$ ) or nifedipine (average concentration, above $5.8 \times 10^{-7} \mathrm{M}$ ) also inhibited the generation of $\mathrm{RC}$ together with the reduction in tension by about $40 \%$ of control tension (Fig. 6). A restoration of RC after washing out the drug, however, took a long time, more than $30 \mathrm{~min}$. A manual increase in stretch tension failed to 


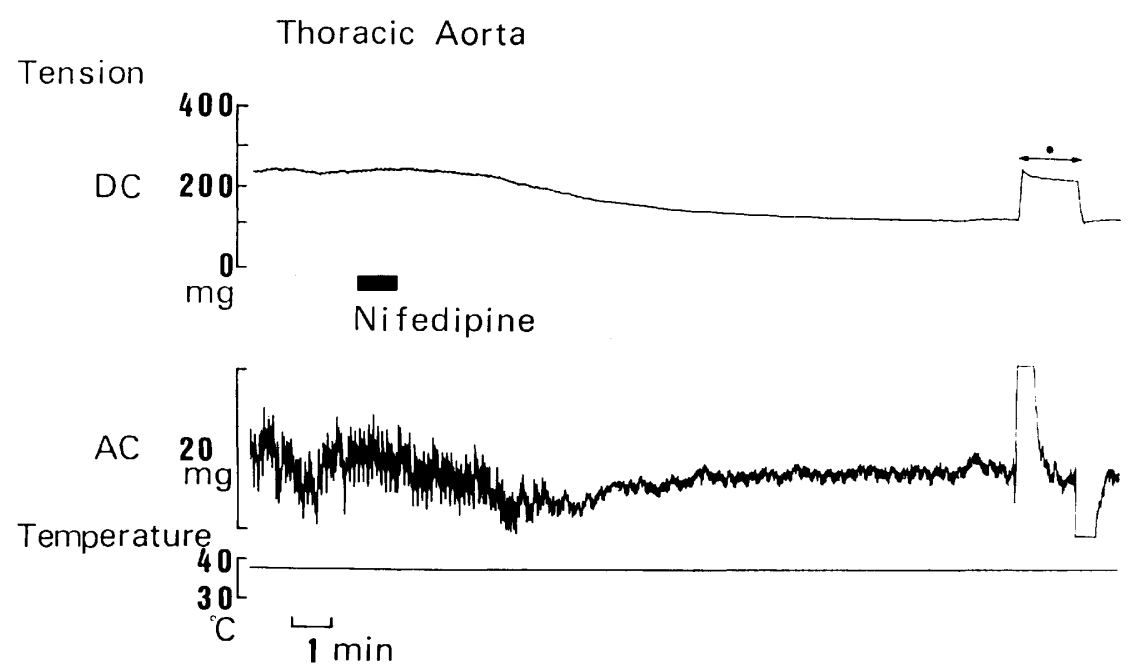

Fig. 6. Effect of Ca blocker (nifedipine) on spontaneous rhythmic contraction in the rat thoracic aorta. The presence of rhythmic contraction is seen in the AC mode registration. Nifedipine was injected for a period indicated by the bar (approximate mean concentration in the incubation chamber, $5.8 \times 10^{-7} \mathrm{M}$ ). After application of nifedipine, rhythmic contraction was not initiated by a passive increase in the stretch tension (arrow with dot). The AC mode registration is partly saturated on a pen recorder during artifactual changes in tension.

evoke the $\mathrm{RC}$ in $\mathrm{Ca}-$ free condition or during applicating the Ca-entry blocker (Figs. 5 and 6 , dot). The inhibition of $\mathrm{RC}$, therefore, seems to have reduced the vascular tension but not vice versa. The small irregular changes in tension, which remained during cessation of $\mathrm{RC}$, were not abolished by prolonged exposure to $\mathrm{Ca}$-free solution or to Ca-entry blockers in high concentrations.

\section{Effects of adrenergic blockers}

The effects of various adrenergic blockers (phentolamine, prazosin, yohimbine, and propranolol) on the RC were examined. An $\alpha$-blocker phentolamine, when applied on the preparation in the average concentration of $1.0 \times 10^{-7}-1.0 \times 10^{-5} \mathrm{M}$, affected neither the frequency and amplitude of RC nor the vascular tension; while phentolamine in concentration, above $1.6 \times 10^{-4} \mathrm{M}$, clearly inhibited the RC and reduced the vascular tension. This inhibitory effect, however, seemed to be due to nonspecific membrane action of the drug in an extremely high concentration. Applications of other adrenergic blockers (prazosin, $1.0 \times 10^{-6}-1.0 \times 10^{-4} \mathrm{M}$ (Fig. 7); yohimbine, $1.0 \times 10^{-6}-1.0 \times 10^{-5} \mathrm{M}$; propranolol, $1.0 \times 10^{-6}-1.0 \times 10^{-5} \mathrm{M}$ ) also had no obvious effects on the $\mathrm{RC}$ and tension. These results suggest that the adrenergic mechanism being activated by endogenous catecholamine is not involved in the initiation of $\mathrm{RC}$ in the isolated rat aorta. 


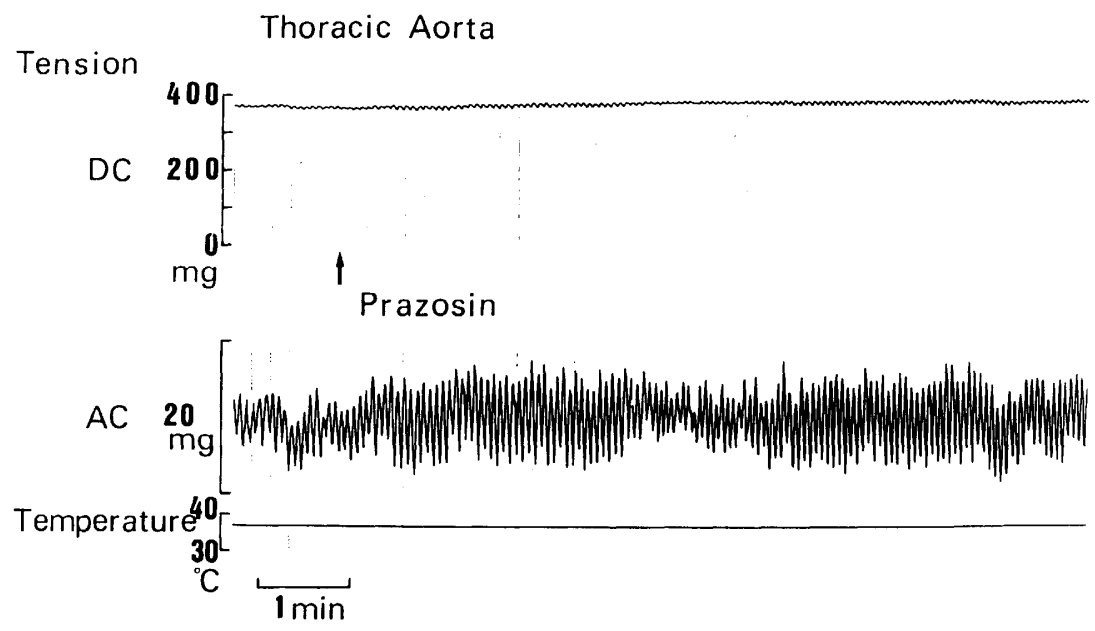

Fig. 7. Effect of $\alpha$-adrenergic blocker (prazosin) on spontaneous rhythmic contraction in the thoracic aorta. The arrow indicates the starting time when the solution containing a given concentration of prazosin $\left(1.0 \times 10^{-6} \mathrm{M}\right)$ was superfused continuously. Prazosin affected neither the frequency nor the amplitude of rhythmic contraction.

\section{DISCUSSION}

In the present experiment spontaneous contractions were observed consistently in the isolated rat aorta even in the absence of external stimuli. This contrasts to the previous observations that rhythmic mechanical activities of large elastic arteries, especially those of the aorta, are seen only occasionally and are brought about by applications of norepinephrine and high potassium or by low Mg solution (BIAMINO and Kruckenberg, 1969; Altura and Altura, 1974; Altura et al., 1980). In the present experiment, we recorded not only the baseline tension of vascular preparation with a conventional low gain DC mode but also changes in tension with a high gain amplification of tension signals in the AC mode. As described previously (MASUDA et al., 1982), the latter method of tension registration may be effective in detecting the spontaneous mechanical activity if the amplitude of changes in vascular tension is small. Another possibility to explain the frequent occurrence of $\mathrm{RC}$ in the present study may be that the smooth muscle cells of the rat aorta are inherently characteristic of generating spontaneous contraction more than that of other mammalian species. This possibility remains to be verified. In the rat aorta, the smooth muscle cells are arranged diagonally in parallel array, but successive layers are oriented in different directions, i.e., high pitch or almost longitudinal and low pitch or almost transverse arrangements (PEASE, 1962; RHODIN, 1980). Since our preparations were made by transverse section, the $\mathrm{RC}$ was presumably generated in the muscle layer that was oriented in more or less transverse direction. 
The $\mathrm{RC}$ was relatively small in amplitude and was accompanied by small oscillatory changes in membrane potential. Since slow potential changes recorded in the present study are small in amplitude and lack spike components, it is uncertain whether they are involved in triggering contraction. If this is the case, the cell to cell conduction of such a small depolarization may not spread widely and hence concomitant recruitment of contracting smooth muscle cells may be limited, initiating a relatively small rhythmic contraction. A complete synchronization between each contraction and slow potential change in the recorded cell was not always seen. This fact suggests that the RC may be initiated by membrane depolarization not only from a single pacemaker but also from a few but multiple pacemaker cells. The frequency of RC was not increased clearly in the static phase of passive stretch although in some cases there was a slight and transient increase in frequency in the dynamic phase of stretch. Johansson and Mellander (1975) showed that the portal mesenteric vein exhibited a significant increase in the frequency of spontaneous action potentials only during the dynamic phase of stretch. A rapid stretch of the preparation did not influence the membrane potential in the present study. The lack of effect of stretch may be due to the stretch being applied only for a short period (within 2-3 s) or to the extent of stretch being small, or to both. Since effects of variation in the passive stretch on the activity of vascular smooth muscle are of physiological significance, influences of stretch on the membrane potential of large elastic artery should be more precisely examined in further experiments. The average membrane potential of cell in rat aorta was smaller than that reported in the rabbit large arteries (MeKATA, 1974). We could not, however, find any comparable data for the rat large arteries.

The frequency of RC was decreased by low temperature. Generally, low temperature inhibits the initiation of spontaneous activity in vascular smooth muscles and subsequently reduces the vascular tension in the deep vessels (Keatinge, 1964; VAnhoutte and Lorenz, 1970; Vanhoutte, 1980). The cessation of $\mathrm{RC}$ by $\mathrm{Ca}$-free solution or by $\mathrm{Ca}$ blocking agents means that the presence of extracellular $\mathrm{Ca}$ and/or its entry into the cell is necessary for the generation of spontaneous electrical activities or for the excitation (depolarization)-contraction coupling of smooth muscles. Rhythmic contractions of the rat portal vein and aorta have been shown to be blocked by Ca-free solution or by Ca-entry blockers (Golenhofen and Weston, 1976; Altura et al., 1980; Vanhoutte, 1981). Various adrenergic blockers were ineffective in suppressing the spontaneous RC. Furthermore, the aortic preparation isolated from the reserpine-pretreated rat showed also a typical RC (our unpublished observation). Thus the activation of vascular smooth muscles by catecholamines which may be released endogenously from sympathetic nerve terminals, if any, is not essential for the generation of spontaneous RC in the rat aorta. PATIL et al. (1972) described the absence of the adrenergic nerve terminals in the rat aorta.

After the RC had been inhibited by a low temperature (below $30^{\circ} \mathrm{C}$ ) or by Cafree solution, small irregular changes in tension remained in every preparation. This 
small mechanical activity was suppressed by an extremely low temperature (below $10^{\circ} \mathrm{C}$ ) but not by $\mathrm{Ca}$ blockers. The nature of this remaining activity is not clear. It may represent a compound form of spontaneous random contractions which occurs in a very localized group of smooth muscle cells because its amplitude was extremely small and its frequency was irregular. There is also a possibility that the small irregular activity represents the mechanical movements of structures other than the smooth muscle, such as the endothelial cells. Recent evidence suggests the ability of vascular endothelial cells to contract (BEvan et al., 1980).

An inhibition of $\mathrm{RC}$ by low temperature, $\mathrm{Ca}$-free solution or by $\mathrm{Ca}$ blockers reduced the baseline vascular tension, which suggests that the generation of RC may increase the vascular tension in the large artery.

The present results do not provide any evidence that the RC occurs spontaneously in in situ preparation and that it influences the vascular hemodynamics in the large artery. The presence of spontaneous contraction in situ aorta has been recently shown by MANGEL et al. (1981). Possible effects of spontaneous RC on the distensibility of elastic artery (Windkessel vessels) should be examined in further experiments using a more appropriate preparation such as a cylindrical segment of conduit artery.

\section{REFERENCES}

Altura, B. M. and Altura, B. T. (1974) Magnesium and contraction of arterial smooth muscle. Microvasc. Res., 7: 145-155.

Altura, B. M., Altura, B. T., and Carella, A. (1980) Effects of ketamine on vascular smooth muscle function. Br. J. Pharmacol., 70: 257-267.

Bevan, J. A., Bevan, R. D., and Duckles, S. P. (1980) Adrenergic regulation of vascular smooth muscle. In: Handbook of Physiology, Sec. 2, The Cardiovascular System, ed. by Bohr, D. F., Somlyo, A. P., and Sparks, H., Am. Physiol. Soc., Bethesda, Maryland, Vol. II, pp. 515-566.

Biamino, G. and KrUCKenBerg, P. (1969) Synchronization and conduction of excitation in the rat aorta. Am. J. Physiol., 217: 376-382.

FUNAKI, S. and BoHR, D. F. (1964) Electrical and mechanical activity of isolated vascular smooth muscle of the rat. Nature, 203: 192-194.

Golenhofen, G. and Weston, H. (1976) Differentiation of calcium activation system in vascular smooth muscle. In: Ionic Actions on Vascular Smooth Muscles, ed. by BETZ, E., Springer-Verlag, Berlin, Heidelberg, New York, pp. 21-25.

JoHANSSON, B. and BoHR, D. F. (1966) Rhythmic activity in smooth muscle from small subcutaneous arteries. Am. J. Physiol., 210: 801-806.

Johansson, B. and Mellander, S. (1975) Static and dynamic components in the vascular myogenic response to passive changes in length as revealed by electrical and mechanical recordings from the rat portal vein. Circ. Res., 36: 76-83.

KeAtinge, W. R. (1964) Mechanism of adrenergic stimulation of mammalian arteries and its failure at low temperatures. J. Physiol. (Lond.), 174: 184-205.

Mangel, A., Faim, M., and van Breemen, C. (1981) Rhythmic contractile activity of the in vivo rabbit aorta. Nature, 289: 692-694.

Masuda, Y., OKuI, K., and FuKuda, Y. (1982) Fine spontaneous contractions of the 
arterial wall of the rat in vitro. Jpn. J. Physiol., 32: 453-457.

Mekata, F. (1974) Current spread in the smooth muscle of the rabbit aorta. J. Physiol. (Lond.), 242: 143-155.

Patil, P. N., Fudge, K., and Jacobowitz, D. (1972) Steric aspect of adrenergic drugs. XVIII, $\alpha$-adrenergic receptors of mammalian aorta. Eur. J. Pharmacol., 19: 79-87.

Pease, D. C. (1962) Arterial and arteriolar system. Microscopic and submicroscopic anatomy. In: Blood Vessels and Lymphatics. ed. by Abramson, D. I., Academic Press, London and New York, pp. 12-25.

Rhodin, J. A. G. (1980) Architecture of vessel wall. In: Handbook of Physiology, Sec. 2, The Cardiovascular System, ed. by Bohr, D. F., Somlyo, A. P., and Sparks, H., Am. Physiol. Soc., Bethesda, Maryland, Vol. II, pp. 1-31.

RoddiE, I. C. and ScotT, A. C. (1969) Some differences in the responses of the arterial and venous smooth muscle to temperature changes. $J$. Physiol. (Lond.), 201: 43-45.

VAnhoutte, P. M. (1980) Physical factor for regulation. In: Handbook of Physiology, Sec. 2, The Cardiovascular System, ed. by Bohr, D. F., Somlyo, A. P., and Sparks, H., Am. Physiol. Soc., Bethesda, Maryland, Vol. II, pp. 443-474.

Vanhoutte, P. M. (1981) Differential effects of calcium entry blockers on vascular smooth muscles. In: New Perspectives on Calcium Antagonists, ed. by Weiss, G. B., Am. Physiol. Soc., Bethesda, Maryland, pp. 109-121.

Vanhoutte, P. M. and Lorenz, R. R. (1970) Effect of temperature on reactivity of saphenous, mesenteric and femoral veins of the dog. Am. J. Physiol., 218: 1746-1750. 